\title{
OPEN Discovery of actinomycin $L$, a new member of the actinomycin family of antibiotics
}

\begin{abstract}
Nataliia V. Machushynets ${ }^{1}$, Somayah S. Elsayed ${ }^{1}$, Chao Du $^{1}$, Maxime A. Siegler ${ }^{2}$, Mercedes de la $\mathrm{Cruz}^{3}$, Olga Genilloud ${ }^{3}$, Thomas Hankemeier ${ }^{4}$ \& Gilles P. van Wezel ${ }^{1 凶}$

Streptomycetes are major producers of bioactive natural products, including the majority of the naturally produced antibiotics. While much of the low-hanging fruit has been discovered, it is predicted that less than $5 \%$ of the chemical space of natural products has been mined. Here, we describe the discovery of the novel actinomycins $L_{1}$ and $L_{2}$ produced by Streptomyces sp. MBT27, via application of metabolic analysis and molecular networking. Actinomycins $L_{1}$ and $L_{2}$ are diastereomers, and the structure of actinomycin $L_{2}$ was resolved using NMR and single crystal $X$-ray crystallography. Actinomycin $L$ is formed via spirolinkage of anthranilamide to the 4-oxoproline moiety of actinomycin $X_{2}$, prior to the condensation of the actinomycin halves. Such a structural feature has not previously been identified in naturally occurring actinomycins. Adding anthranilamide to cultures of the actinomycin $X_{2}$ producer Streptomyces antibioticus, which has the same biosynthetic gene cluster as Streptomyces sp. MBT27, resulted in the production of actinomycin L. This supports a biosynthetic pathway whereby actinomycin $L$ is produced from two distinct metabolic routes, namely those for actinomycin $X_{2}$ and for anthranilamide. Actinomycins $L_{1}$ and $L_{2}$ showed significant antimicrobial activity against Gram-positive bacteria. Our work shows how new molecules can still be identified even in the oldest of natural product families.
\end{abstract}

Considering the emerging crisis of antibiotic resistance that spreads among bacterial pathogens and increasing incidence of cancer, the search for new, efficient and less toxic drugs remains a priority ${ }^{1,2}$. Actinobacteria have been the source for the majority of the antibiotics in use today ${ }^{3,4}$. Of the Actinobacteria, members of the genus Streptomyces produce over half of all currently characterized antibiotics ${ }^{3}$. Genome sequencing revealed that Actinobacteria have much more biosynthetic potential to produce bioactive molecules than originally anticipated, with even the model organisms harbouring many so-called cryptic or silent biosynthetic gene clusters (BGCs) that specify yet unknown compound $s^{5-7}$. Triggering the expression of silent BGCs by genetic and cultivationbased techniques should facilitate unlocking this yet unexplored chemical diversity, allowing the discovery of novel molecules ${ }^{8,9}$. This strategy relies on altering the regulatory networks of the producing organism in response to fluctuating culturing conditions, such as carbon, nitrogen or phosphate concentration ${ }^{10-12}$. Manipulation of fermentation conditions of promising producer strains, known as "one strain many compounds" (OSMAC) approach, is effective in enhancing secondary metabolites production ${ }^{13,14}$. Novel secondary metabolites have been discovered via modification of cultivation parameters, including nutrients ${ }^{15,16}$, and addition of chemical elicitors ${ }^{17,18}$.

Metabolic profiling of crude extracts obtained under different growth conditions represents a challenging analytical task since these mixtures are composed of hundreds of natural products. Therefore, metabolomics, particularly those based on mass spectrometry (MS), became more and more valuable and greatly increased the efficiency of such screenings ${ }^{19}$. Supervised statistical methods are able to classify a response like a biological activity, and to determine the most discriminant metabolite(s) related to such response ${ }^{20}$. Moreover, simultaneous dereplication of differentially expressed compounds is implemented into the drug-discovery pipelines in order to avoid rediscovery of already known compounds ${ }^{21}$. MS-based metabolomics provides important information on the distribution of the metabolites that are present in complex mixtures, but the identification of their structures is complicated. For this purpose, the Global Natural Products Social Molecular Networking (GNPS) platform

\footnotetext{
${ }^{1}$ Molecular Biotechnology, Institute of Biology, Leiden University, Sylviusweg 72, 2333 BE Leiden, The Netherlands. 'Department of Chemistry, The Johns Hopkins University, 3400 North Charles Street, Baltimore,

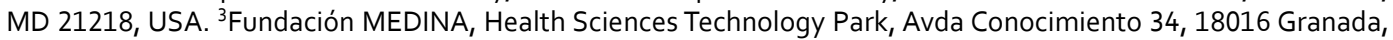
Spain. ${ }^{2}$ Leiden Academic Centre for Drug Research (LACDR), Leiden University, Einsteinweg 55, 2333 CC Leiden, The Netherlands. ${ }^{\varpi}$ email: g.wezel@biology.leidenuniv.nl
} 

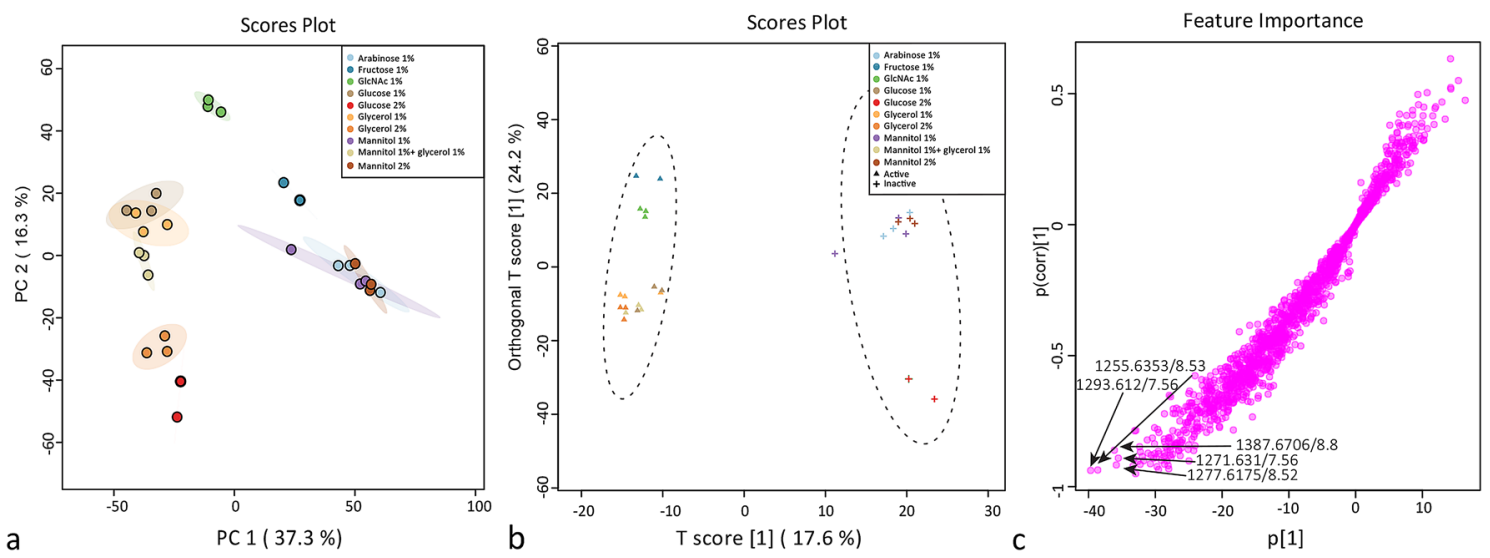

Figure 1. Differential production of metabolites depending on the carbon source. (a) PCA score plot of Streptomyces sp. MBT27 metabolites produced in cultures with different carbon sources, namely, $1 \%$ arabinose, $1 \%$ fructose, $1 \%$ GlcNAc, $1 \%$ glucose, $2 \%$ glucose, $1 \%$ glycerol, $2 \%$ glycerol, $1 \%$ mannitol, $1 \%$ mannitol $+1 \%$ glycerol and $2 \%$ mannitol (\%ages in w/v). (b) OPLS-DA score plot. Triangles and crosses represent samples of active and inactive groups respectively, circular areas represent the $95 \%$ confidence region of each group. (c) OPLS-DA loadings S-plot. Arrows indicate the most discriminative features that positively correlate with the active groups.

was developed, applying both molecular networking and automated searches of tandem mass spectrometry (MS/ MS) fragmentation spectra against spectral libraries, to identify structural relationships between metabolites ${ }^{22,23}$. This greatly facilitates the annotation and dereplication of known molecules.

Actinomycin is a DNA-targeting antibiotic and anticancer compound discovered in 1940 by Waksman \& Woodruff, and in fact the first antibiotic that was isolated from an actinobacterium ${ }^{24}$. Actinomycins are produced by various Streptomyces strains and are composed of a chromophore group and two pentapeptide chains with a variable composition of amino acids ${ }^{25}$ (Fig. S1). Actinomycins D, $\mathrm{X}_{0 \beta}$ and $\mathrm{X}_{2}$ are usually simultaneously produced and differ from each other by substitutions on the proline residue in their pentapeptide lactone rings, while members of the actinomycin $\mathrm{C}$ complex vary in their $\mathrm{D}$-valine residues ${ }^{26}$. The pentapeptide precursors are biosynthesized by a non-ribosomal peptide synthetase (NRPS) assembly line, and actinomycins are formed through oxidative condensation of two 3-hydroxy-4-methylanthranilic acid (4-MHA) pentapeptide lactones (PPLs) ${ }^{27}$.

In this work we report the discovery of new actinomycin analogues, actinomycin $\mathrm{L}_{1}$ and $\mathrm{L}_{2}$, from the extracts of Streptomyces sp. MBT27. Multivariate data analysis combined with molecular networking indicated that the antimicrobial activity of the extracts correlated with novel actinomycins $\mathrm{L}_{1}$ and $\mathrm{L}_{2}$ and known actinomycins $\mathrm{D}$, $\mathrm{X}_{0 \beta}$ and $\mathrm{X}_{2}$. NMR and single crystal $\mathrm{X}$-ray crystallography revealed that an anthranilamide moiety was linked through a spiro-center to a proline residue in the structure of actinomycins $\mathrm{L}_{1}$ and $\mathrm{L}_{2}$. Such a structural feature has not previously been identified in naturally occurring actinomycins.

\section{Results}

The influence of carbon sources on bioactivity and actinomycin production. Streptomyces sp. MBT27 is a gifted natural product producer that was isolated from Qinling mountains in China, with potent antibacterial activity against various MDR (multi-drug resistant) bacteria ${ }^{28}$. We previously showed that the strain among others produces the novel quinazolinones A and $\mathrm{B}^{29}$. To investigate the antibiotic activity of Streptomyces sp. MBT27 the strain was fermented in minimal medium (MM) with either of the following carbon sources (percentages in w/v): $1 \%$ of both mannitol and glycerol, $1 \%$ mannitol, $2 \%$ mannitol, $1 \%$ glycerol, $2 \%$ glycerol, $1 \%$ glucose, $2 \%$ glucose, $1 \%$ fructose, $1 \%$ arabinose, or $1 \% N$-acetylglucosamine (GlcNAc). Supernatants of Streptomyces sp. MBT27 cultures were extracted with ethyl acetate and bioactivity assays were performed against Bacillus subtilis 168. Interestingly, the carbon sources had a huge effect on the antimicrobial activity (Fig. S2). Particularly strong antimicrobial activity was observed when the culture medium was supplemented with glycerol + mannitol, glucose 1\%, glycerol, fructose or GlcNAc; as compared to when mannitol or arabinose were used as the carbon sources.

In order to investigate the metabolic differences due to nutritional supplementation and correlate that to the antimicrobial activity, LC-MS-based metabolomics was performed. Initially, the LC-MS data were explored by unsupervised Principal Component Analysis (PCA). The first two PCs accounted for 37\% and 16\%, respectively, of the total data variation. PCA analysis failed to show significant metabolic separation in relation to the observed bioactivity (Fig. 1a). The supervised Orthogonal Partial Least Squares Discriminant Analysis (OPLS-DA) was then applied to discriminate the samples based on their ability to inhibit B. subtilis (Fig. 1b). The cross-validation metrics of the model $\left(R^{2} Y=0.748\right.$ and $\left.Q^{2} Y=0.676\right)$ indicated that the model has a good reliability and ability of prediction. A permutation test was performed $(n=100)$ and the resulting $R^{2} Y$ and $Q^{2} Y$ values were significantly lower ( $p$ values $<0.01$ for both), which indicated that there was no overfitting in the model ${ }^{30}$ (Fig. S3). The OPLS-DA loadings S-plot revealed the most discriminative features between active and inactive groups (Fig. 1c). 


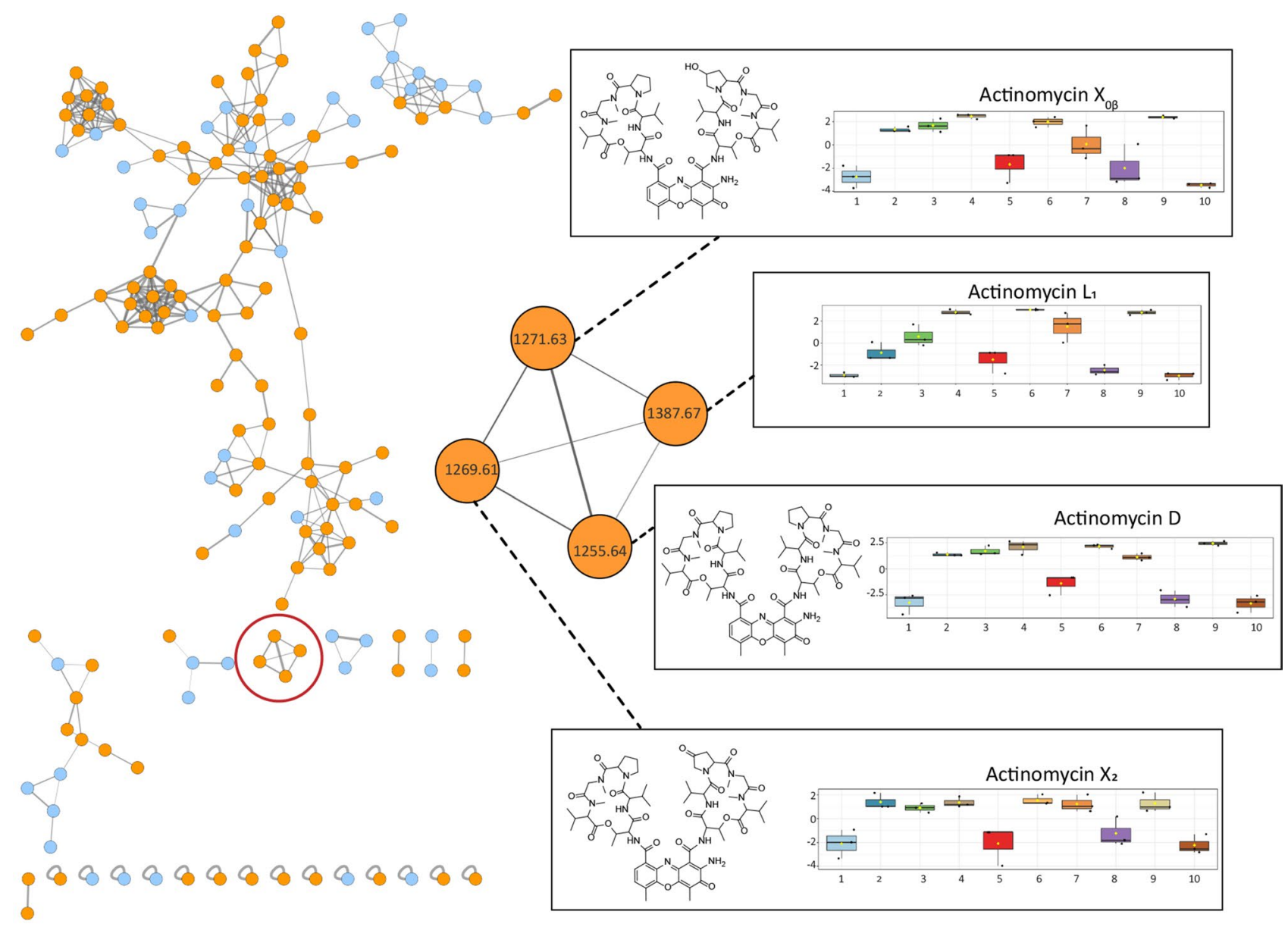

808080808080808

Figure 2. GNPS molecular network of the ions detected in the crude extract of Streptomyces sp. MBT27. Cultures were grown for seven days in MM with $1 \%$ glycerol. Orange nodes represent ions of the metabolites produced by Streptomyces sp. MBT27, while blue nodes represent those of the media components. The actinomycin spectral family is enlarged. Results of ANOVA statistical analysis were mapped onto the molecular network to illustrate the differential production of actinomycin cluster members under various growth conditions. Box plots represent relative intensities of actinomycins $\mathrm{X}_{2}, \mathrm{X}_{0 \beta}$, and $\mathrm{D}$ after $\log$ transformation and pareto scaling; together with a compound with an $m / z$ value of 1387.67 , in cultures grown in MM with the following carbon sources: $1.1 \%$ arabinose; $2.1 \%$ fructose; $3.1 \%$ GlcNAc; $4.1 \%$ glucose; $5.2 \%$ glucose; $6.1 \%$ glycerol; 7. 2\% glycerol; 8. 1\% mannitol; 9. 1\% mannitol $+1 \%$ glycerol; $10.2 \%$ mannitol (\%ages in w/v).

The mass features that correlated best to the bioactivity were $\mathrm{m} / z 1387.6706(8.8 \mathrm{~min}), \mathrm{m} / z 1255.6353$ (8.53 $\mathrm{min}$ ), $m / z 1277.6175$ (8.52 $\mathrm{min}$ ), $m / z 1271.651$ (7.56 min) and $m / z 1293.612$ (7.56 min) (Fig. 1c). Dereplication of those features was performed through comparison of the UV spectra, accurate masses, isotope distribution and fragmentation patterns obtained in MS/MS analysis against the chemistry databases Reaxys, ChemSpider, and the microbial natural products database Antibase ${ }^{31}$. This allowed us to annotate the mass features with $\mathrm{m} / z 1255.6353$ and 1277.6175 as the $[\mathrm{M}+\mathrm{H}]^{+}$and $[\mathrm{M}+\mathrm{Na}]^{+}$adduct ions of actinomycin $\mathrm{D}$, respectively, while the mass features with $m / z 1271.651$ and 1293.612 were annotated as $[\mathrm{M}+\mathrm{H}]^{+}$and $[\mathrm{M}+\mathrm{Na}]^{+}$adduct ions of actinomycin $\mathrm{X}_{0 \beta}$, respectively ${ }^{27}$. However, the mass feature with an $m / z$ value of $1387.6706[\mathrm{M}+\mathrm{H}]^{+}$could not be matched to any of the previously reported microbial natural products.

Global Natural Product Social (GNPS) molecular networking ${ }^{23}$ was subsequently employed to detect MS/ MS-based structural relatedness among features in an automated manner. The web-based platform generates a molecular network wherein features with related scaffolds cluster together. Cytoscape 3.7.2 was used for visualization of the generated molecular networks ${ }^{32}$. A network representing the ions detected in the crude extract of Streptomyces sp. MBT27 grown with $1 \%$ glycerol was constructed, revealing 172 nodes clustered in 10 spectral families (Fig. 2). The molecular network revealed an actinomycin spectral family containing actinomycin D, $\mathrm{X}_{2}$ and $\mathrm{X}_{0 \beta}$. Moreover, the same spectral family included a yet unidentified compound with $\mathrm{m} / z 1387.67$. It was closely connected (cosine score $>0.7$ ) to the known actinomycins, suggesting that the molecule was a novel actinomycin. Statistical analysis showed that the extracts with stronger antimicrobial activity contained higher concentrations of actinomycins $\mathrm{X}_{2}, \mathrm{X}_{0 \beta}$, D and the new compound, in comparison with the less active ones (ANOVA, $p<0.05$; Fig. 2). It is important to note that actinomycins were only detected in the bioactive extracts. 

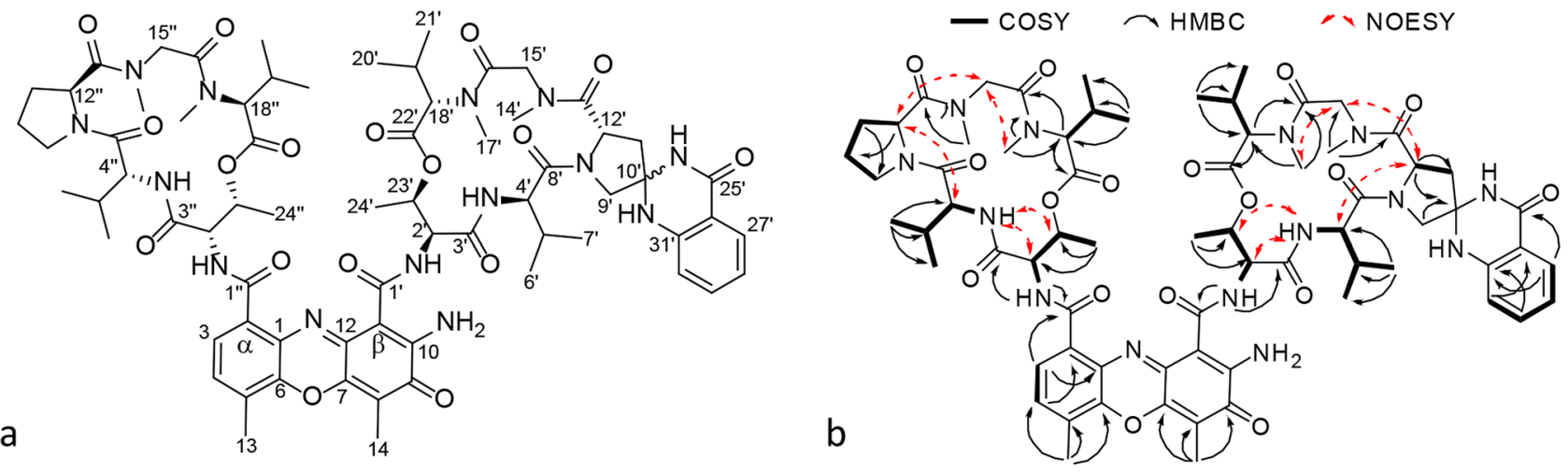

Figure 3. Chemical structures of the new actinomycins. Shown are actinomycin $\mathrm{L}_{1}\left(10^{\prime} S\right)(\mathbf{1})$ and $\mathrm{L}_{2}\left(10^{\prime} R\right)(2)$ (a) and the key COSY, HMBC and NOESY correlations for $\mathbf{1}$ (b).

Large scale fermentation and NMR. To allow identification and structural analysis of the likely novel actinomycin analogue, we performed large-scale fermentation of Streptomyces sp. MBT27 followed by bioactivity guided fractionation. The purification process resulted in the isolation of two compounds (1) and (2), with the same mass (Figs. S4, S5). The NMR spectra of the two compounds were very similar, suggesting that they were diastereomers (Figs. S6-S12). Based on 1D and 2D NMR analysis of 1, together with the molecular formula and degrees of unsaturation dictated by the accurate mass, the structure of the isolated diastereomers was determined as a variant of actinomycin $\mathrm{D}$, whereby an aminal was formed between the amino group of anthranilamide moiety and keto group at the $\gamma$ position of the proline residues (Fig. 3). The prolyl substitution position is the same as that of the hydroxyl and keto groups in actinomycins $\mathrm{X}_{0 \beta}$ and $\mathrm{X}_{2}$, respectively. The new actinomycin analogue was designated actinomycin L (with L standing for Leiden, the city of its discovery).

The second stereoisomer (2) was crystallized successfully. Single-crystal X-ray diffraction confirmed the structure obtained for (1) based on NMR, and established the absolute configuration to be $2^{\prime} S, 2^{\prime \prime} S, 4^{\prime} R, 4^{\prime \prime} R, 10^{\prime} R$, $12^{\prime} S, 12^{\prime \prime} S, 18^{\prime} S, 18^{\prime \prime} S, 23^{\prime} R, 23^{\prime \prime} R$ by anomalous-dispersion effects in diffraction measurements on the crystal (Fig. 4). As the absolute configuration of the amino acid residues in $\mathbf{2}$ was consistent with that of previously reported actinomycins ${ }^{33}$, and considering that the two isomers stemmed from the aminal formation at C-10', compound $\mathbf{1}$ is inevitably the $10^{\prime} \mathrm{S}$ isomer of actinomycin L.

Biosynthesis of actinomycin L. Actinomycins D (or $\mathrm{X}_{1}$ ), $\mathrm{X}_{2}$, and $\mathrm{X}_{0 \beta}$ detected in the extracts of Streptomyces sp. MBT27 are members of the actinomycin $\mathrm{X}$ complex. Recently it was shown that actinomycins $\mathrm{X}_{0 \beta}$ and $\mathrm{X}_{2}$ are formed through the sequential oxidation of the $\gamma$-prolyl carbon by the cytochrome P450 enzyme $\mathrm{saAcmM}^{34,35}$. Based on its structure, actinomycin $\mathrm{L}$ is most likely formed through an aminalization reaction between the two amino groups of anthranilamide and the $\gamma$-keto group on the proline residue of actinomycin $\mathrm{X}_{2}$. Accordingly, its production should be arrested when one of the precursors is not available. Interestingly, Streptomyces sp. MBT27 produced actinomycin L in very low amounts when grown with fructose (1\% w/v) as the sole carbon source. Moreover, ANOVA statistical analysis showed that anthranilamide was produced in equally low amounts under the same growth conditions (ANOVA, $p<0.05$; Fig. 5). Under conditions where Streptomyces sp. MBT27 produced actinomycin L, namely when grown in MM with 1\% GlcNAc, $1 \%$ glucose, $1 \%$ glycerol, $2 \%$ glycerol or in $1 \%$ mannitol $+1 \%$ glycerol, the strain invariably produced both actinomycin $\mathrm{X}_{2}$ and anthranilamide. However, under conditions where actinomycin $\mathrm{X}_{2}$ was produced but not anthranilamide, the strain failed to produce actinomycin L (Fig. 5).

We therefore wondered if anthranilamide may be a precursor for the biosynthesis of actinomycin L. To test this hypothesis, we performed a feeding experiment, whereby anthranilamide was added to cultures of Streptomyces sp. MBT27 grown in MM with 1\% fructose, where virtually no actinomycin L was produced. Analysis of the supernatant of the cultures via LC-MS revealed that actinomycin L was readily produced when anthranilamide was added, but not without it (Fig. 6a). This strongly suggested that anthranilamide is required for the production of actinomycin L. However, extracts of Streptomyces sp. MBT27 fermented with 1\% fructose and additional anthranilic acid contained both anthranilamide and actinomycin L (Fig. 6a). This suggests that indeed anthranilic acid is converted into anthranilamide, which in turn is incorporated into actinomycin $\mathrm{L}$.

In order to unambiguously verify that actinomycin $\mathrm{L}$ was the product of anthranilamide and actinomycin $\mathrm{X}_{2}$, we conducted another biotransformation experiment, now feeding anthranilamide to S. antibioticus IMRU 3720, which is a known producer of actinomycins $\mathrm{X}_{2}$ and $\mathrm{X}_{0 \beta}$, but fails to produce actinomycin $\mathrm{L}$ under any condition tested. In line with our hypothesis, S. antibioticus IMRU 3720 also failed to produce anthranilamide under any of the growth conditions (Fig. S17). Excitingly, LC-MS analysis revealed the production of actinomycin L by S. antibioticus IMRU 3720 when anthranilamide was fed to the cultures, but never without anthranilamide (Fig. 6b). This validates the concept that anthranilamide is a key precursor of actinomycin L. Conversely, when anthranilic acid instead of anthranilamide was added to cultures of S. antibioticus IMRU 3720, we failed to detect actinomycin $\mathrm{L}$ and anthranilamide (Fig. 6b).

The oxidation of the proline residue in actinomycins $\mathrm{X}_{0 \beta}$ and $\mathrm{X}_{2}$ occurs following the formation of the two halves of actinomycin, known as 4-MHA PPLs, and prior to the condensation of these halves to form 


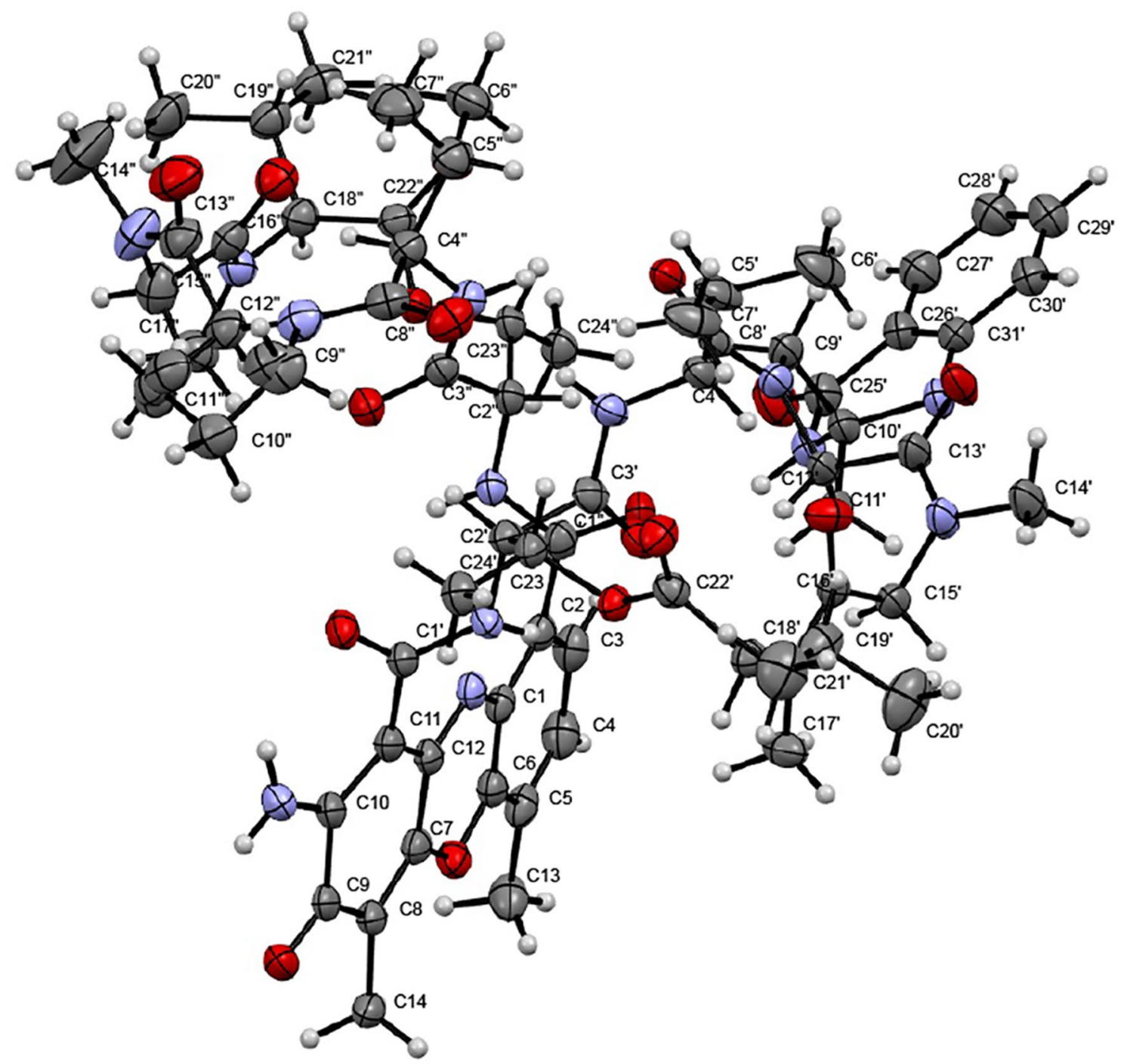

Figure 4. X-ray ORTEP drawing of the crystal structure of compound 2.

actinomycin $^{34}$. Taking this into account we reasoned that anthranilamide should be incorporated into the actinomycin halves prior to condensation. To check this, 3-hydroxy-4-methylbenzoic acid (4-MHB) was added to cultures of Streptomyces sp. MBT27 and of S. antibioticus IMRU 3720. 4-MHB is a structural analogue of 4-MHA that replaces 4-MHA as a starter unit in the nonribosomal assembly of the actinomycin halves ${ }^{34}$. When 4-MHB replaces 4-MHA, 4-MHB containing PPLs accumulate, because they cannot react with each other to give a phenoxazinone ring, as is the case with 4-MHA PPLs ${ }^{36}$. LC-MS analysis of the 4-MHB-supplemented extracts showed the appearance of the previously reported 4-MHB-containing pentapeptide lactones PPL 1 , PPL 0 , and PPL 2, and new PPL, designated as PPL 3 (Figs. S18-S21, Table S1). The exact mass and fragmentation pattern of PPL 3 was consistent with a 4-MHB containing PPL wherein an anthranilamide moiety had been attached to the proline residue (Fig. S21).

Taken together, the feeding experiments convincingly show that actinomycin $\mathrm{L}$ is formed through reaction of anthranilamide with the 4-keto group on the proline residue in the pentapeptide lactone. Moreover, results of the feeding experiments with 3-hydroxy-4-methylbenzoic acid show that this reaction occurs prior to the condensation of the pentapeptide lactones into actinomycin L (Fig. 7).

Identification of the actinomycin BGC in Streptomyces sp. MBT27. To characterize the BGC responsible for actinomycin biosynthesis and compare the genes with those found in known actinomycin BGCs, Streptomyces sp. MBT27 was sequenced using the PacBio platform. Assembly of the PacBio reads resulted in two contigs of $8.4 \mathrm{Mb}$ and $0.13 \mathrm{Mb}$ in length. Analysis using AntiSMASH $6^{37}$ readily identified the actinomycin BGC in the $8.4 \mathrm{Mb}$ contig. Comparison to the actinomycin $\mathrm{X}_{2} \mathrm{BGC}$ from $S$. antibioticus showed that all genes were highly conserved between the two clusters (Table S1 and Fig. S22). This strongly suggests that the actinomycin BGC does not specify the observed modifications in the actinomycin structure, and is not responsible for the 


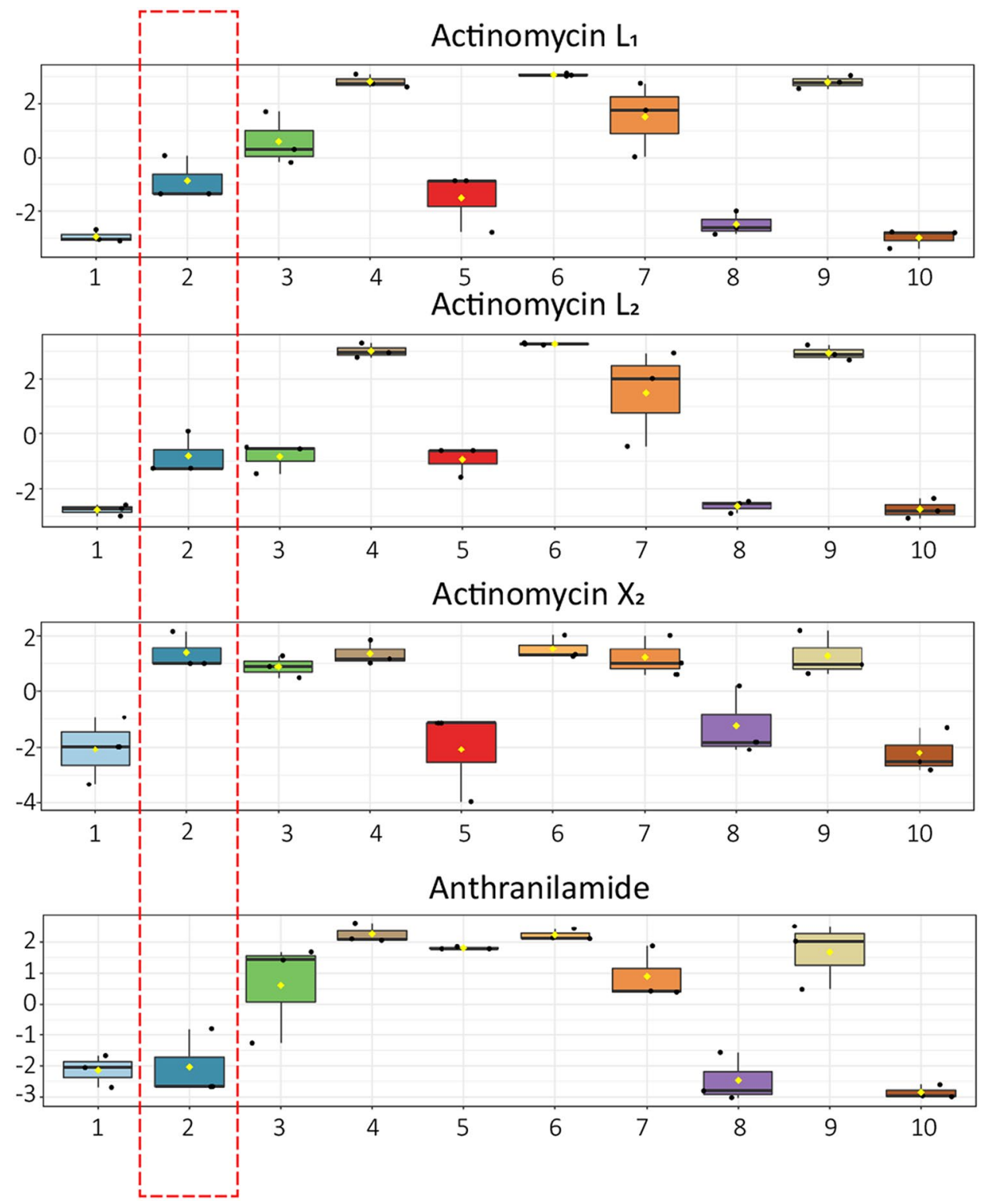

Figure 5. Box plots showing the relative intensities of actinomycin $\mathrm{L}_{1}, \mathrm{~L}_{2}$ and $\mathrm{X}_{2}$ and anthranilamide after log transformation and pareto scaling. Cultures of Streptomyces sp. MBT27 were grown for 7 days in MM with the following carbon sources: $1.1 \%$ arabinose; $2.1 \%$ fructose; $3.1 \%$ GlcNAc; $4.1 \%$ glucose; $5.2 \%$ glucose; $6.1 \%$ glycerol; 7. 2\% glycerol; 8. 1\% mannitol; 9. 1\% mannitol $+1 \%$ glycerol; $10.2 \%$ mannitol (\% ages in w/v). Red box indicates the abundance of actinomycin $\mathrm{L}_{1}, \mathrm{~L}_{2}$ and $\mathrm{X}_{2}$ and anthranilamide in the cultures grown with fructose $(1 \% \mathrm{w} / \mathrm{v})$. Note that Streptomyces sp. MBT27 produced actinomycin L and anthranilamide in very low amounts when fermented with fructose $(1 \% \mathrm{w} / \mathrm{v})$ as the sole carbon source.

production of anthranilamide. We have not yet identified the enzyme for the predicted conversion of anthranilic acid to anthranilamide.

Bioactivity of isolated compounds (MIC). Bioactivity assays were carried out for the actinomycins, to test their ability to act as antibiotics. As expected, the compounds showed selective antibacterial activity against Gram-positive pathogens, and none of the actinomycins presented any activity against E. coli ATCC25922 or K. pneumoniae ATCC700603 (Table 1). All compounds except actinomycin $\mathrm{X}_{0 \mathrm{~B}}$ showed antibacterial activity against Gram-positive bacteria with MIC values ranging from 1 to $16 \mu \mathrm{g} / \mathrm{mL}$. Actinomycin $\mathrm{L}_{1}$ showed somewhat higher bioactivity than actinomycin $\mathrm{L}_{2}$, while both compounds showed slightly higher MICs than actinomycin $\mathrm{D}$ and Actinomycin $\mathrm{X}_{2}$. 
Actinomycin $\mathrm{L}_{1}$

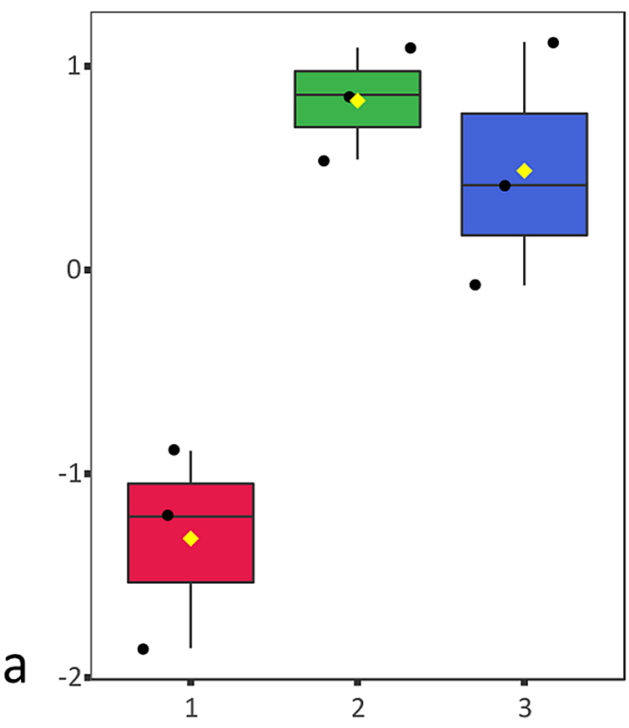

Actinomycin $\mathrm{L}_{1}$

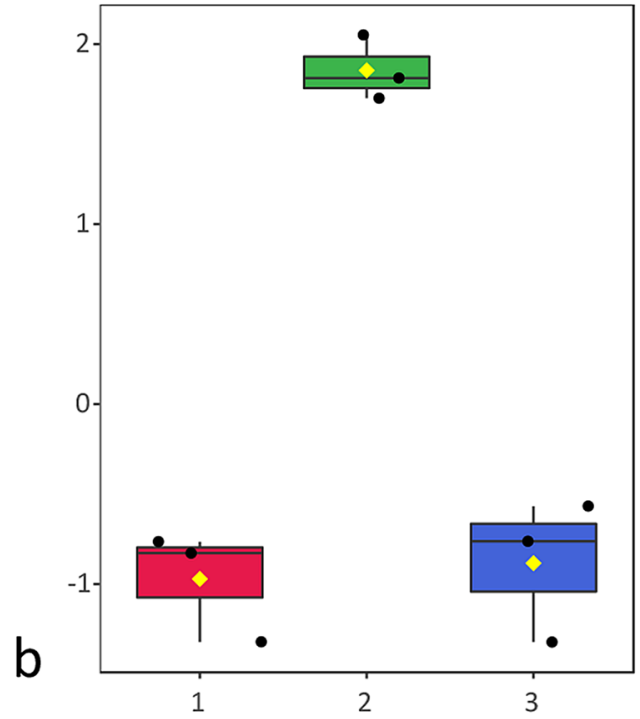

Actinomycin $\mathrm{L}_{2}$

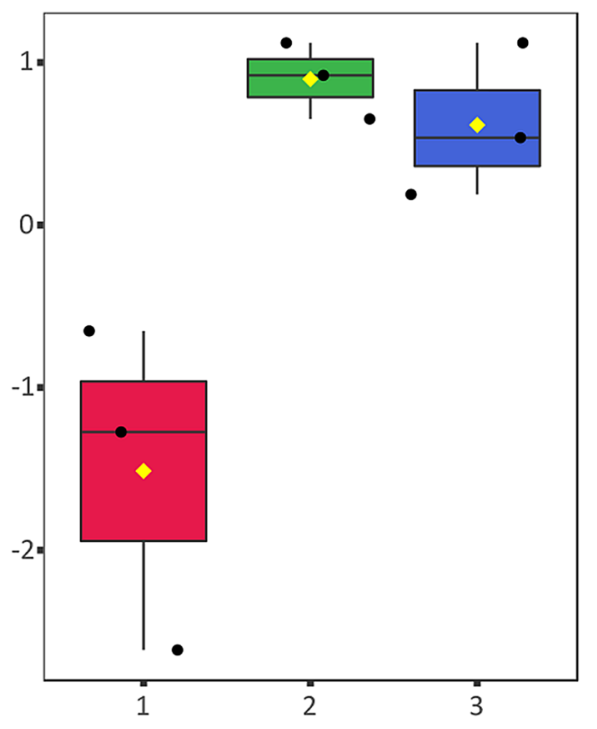

Actinomycin $\mathrm{L}_{2}$

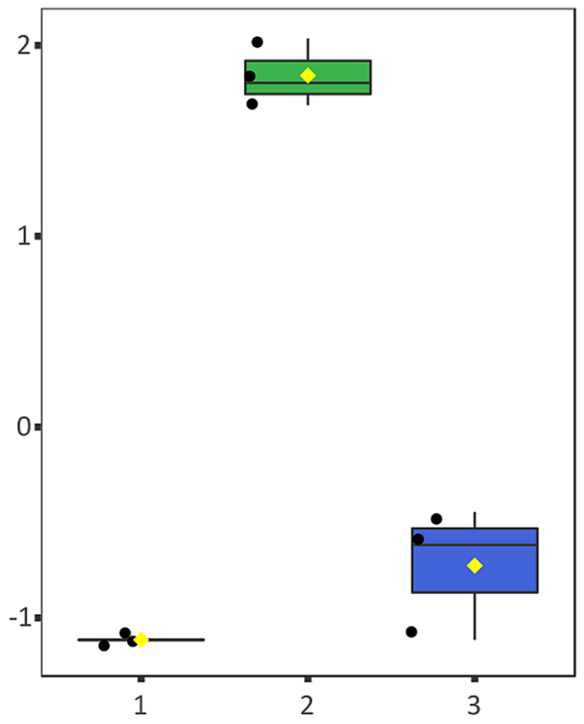

Figure 6. Anthranilamide is required for the biosynthesis of actinomycins $\mathrm{L}_{1}$ and $\mathrm{L}_{2}$. Box plots show the relative intensities of actinomycin $\mathrm{L}_{1}$ and $\mathrm{L}_{2}$ after $\log$ transformation and pareto scaling in the cultures of Streptomyces sp. MBT27 (a) and S. antibioticus (b) fermented for 7 days in MM with fructose (1\% w/v) (1), fed with $0.7 \mathrm{mM}$ anthranilamide (2) and $0.7 \mathrm{mM}$ anthranilic acid (3). Note that $S$. antibioticus produces actinomycin L exclusively in the presence of anthranilamide and not with anthranilic acid; conversely, Streptomyces sp. MBT27 is able to convert anthranilic acid into anthranilamide, enabling the production of actinomycin $\mathrm{L}$.

\section{Discussion}

Actinomycin was the first antibiotic identified in Actinobacteria ${ }^{24}$. The well-established actinomycin structure is composed of a heterocyclic chromophore and two cyclic PPLs. Biosynthetically, PPL is biosynthesized by an NRPS assembly line with the 4-MHA as the initiating unit ${ }^{38,39}$. 4-MHA is derived from 3-hydroxy-4-methylkynurenine (4-MHK), which is formed by methylation of 3-hydroxykynurenine $(3-\mathrm{HK})^{26}$. Our work surprisingly revealed a novel structure within the extensively studied actinomycin family, namely that of actinomycin L, which arises via attachment of an anthranilamide moiety to the $\gamma$-carbon of one of the proline residues through aminal formation. ANOVA statistical analysis proved that production of anthranilamide is the limiting factor in the biosynthesis of actinomycin L. Feeding experiments with anthranilamide suggested that actinomycin $\mathrm{L}$ is formed through the spontaneous reaction of anthranilamide with the 4-oxoproline site of actinomycin $\mathrm{X}_{2}$ prior to the condensation of the two 4-MHA PPLs into actinomycin L. To the best of our knowledge, the attachment of anthranilamide to a 4-oxoproline moiety is a novel observation. 
<smiles>CCNC(=O)C1CC(=O)CN1C(C(C)C)C(C)OC(=O)C(NC(=O)C(NC(=O)C1=C(N)C(=O)C(C)=CC1)C(=O)NC)C(=O)NCC(=O)N(C)C(C(C)C)C(C)C</smiles>

Actinomycin $\mathrm{X}_{2}$ half<smiles>CC1=CCC(C(=O)NC(C(=O)NC(C(=O)NCC(=O)N(C)C(C(=O)OC(C)C(C)C)C(C)C)C(=O)NCC(=O)N2CC3(Nc4ccccc4C(=O)[O-])CNC(=O)C2C3)C(C)C)=C(N)C1=O</smiles><smiles>CC1=CCC(C(=O)NC(C(=O)NC(C(=O)NCC(=O)N2CCCC2C(=O)NCC(=O)N2CCCC2C(C)C)C(C)OC(=O)C(C(C)C)N(C)C)C(C)C)=C(N)C1=O</smiles>

Actinomycin L half<smiles>CC(C)C(NC(=O)C(NC=O)C(C)OC(=O)C(C(C)C)N1CCCC1C(=O)N(C)CC(=O)N1CCCC1C)C(C)C</smiles>

$3 / 2 \mathrm{O}_{2}$<smiles></smiles>

Actinomycin L

Figure 7. Proposed biosynthetic pathway for actinomycin L. We propose that actinomycin L is formed through the reaction of anthranilamide (blue) with the 4-oxoproline moiety of actinomycin $\mathrm{X}_{2}$ prior to the condensation of two 4-MHA PPLs into the actinomycin L.

\begin{tabular}{|c|c|c|c|c|}
\hline Strain & Actinomycin $\mathrm{X}_{2}$ & Actinomycin D & Actinomycin $\mathrm{L}_{1}$ & Actinomycin $\mathrm{L}_{2}$ \\
\hline S. aureus MB5393 (methicillin-resistant) & $2-4 \#$ & $1-2$ & $4-8$ & $8-16$ \\
\hline S. aureus ATCC29213 (methicillin-sensitive) & $1-2$ & $1-2$ & $2-4$ & n.a \\
\hline E. faecium (vancomycin-sentitive) ${ }^{*}$ & $1-2$ & $1-2$ & $4-8$ & $8-16$ \\
\hline E. faecium VanB (vancomycin-resistant)* & $1-2$ & $1-2$ & $4-8$ & $8-16$ \\
\hline S. epidermidis ${ }^{*}$ & $2-4$ & $2-4$ & $4-8$ & n.a \\
\hline E. coli ATCC25922 & $>128$ & $>128$ & $>128$ & n.a \\
\hline K. pneumoniae ATCC700603 & $>128$ & $>128$ & $>128$ & n.a \\
\hline
\end{tabular}

Table 1. Antibacterial activities of compounds expressed as Minimal Inhibitory Concentrations (MIC). $\left({ }^{\star}\right)$, Clinical isolates; n.a.: Data not available. \# all concentrations given in $\mu \mathrm{g} / \mathrm{ml}$.

The actinomycin BGC of Streptomyces sp. MBT27 harbors the same genes as that of S. antibioticus, with high homology between the genes, which strongly suggests that the modification of actinomycin $\mathrm{X}_{2}$ to actinomycin $\mathrm{L}$ is not encoded by the BGC itself. Indeed, we anticipate that anthranilamide is derived from anthranilic acid in Streptomyces sp. MBT27, whereby anthranilic acid in turn is biosynthesized through the shikimate pathway ${ }^{40}$. Anthranilic acid is a commonly produced primary metabolite in Streptomyces, while anthranilamide is less common ${ }^{41-43}$. The actinomycin $\mathrm{X}_{2}$ producer $S$. antibioticus IMRU 3720 fails to convert anthranilic acid into anthranilamide, which explains why actinomycin $\mathrm{L}$ was also not detected in the extracts. However, actinomycin L was produced when we fed cultures of $S$. antibioticus IMRU 3720 with additional anthranilamide, which is fully in line with our proposed biosynthetic pathway. Thus, actinomycin L is an example of a natural product that requires the joining of two separate metabolic pathways, and this is a concept that needs more attention. After all, scientists rely increasingly on heterologous expression and synthetic biology approaches ${ }^{44}$, and these will likely fail if genes are required that do not fall within the main BGC.

The production of actinomycins by Streptomyces spp. is strongly influenced by the carbon source, whereby the preferred carbon source varies from strain to strain ${ }^{45-49}$. D-galactose favors actinomycin production in Streptomyces antibioticus over arabinose, xylose, glucose, fructose and rhamnose $e^{45}$, while glycerol was the optimal carbon source for actinomycin production by S. antibioticus Tü 6040 and S. antibioticus SR $15.4^{46,47}$. In the case of Streptomyces sp. MBT27, growth on MM with glycerol, GlcNAc, fructose and glucose (all 1\% w/v) as sole carbon sources were the best carbon sources to promote the production of actinomycins. However, increasing the glucose concentration to $2 \%$ blocked the production of actinomycins. Glucose was previously reported to repress the transcription of the gene for hydroxykynureninase, which is involved in the formation of the main actinomycin precursor 4-MHA ${ }^{45}$. Importantly, in our experiments the carbon source not only promoted the overall production levels, but also contributed to the chemical diversity of the actinomycins, including the production of actinomycin L. This coincided with the production of anthranilamide, an essential substrate to form this novel actinomycin variant.

In the twenty-first century, genome mining and renewed drug discovery efforts have revealed that Actinobacteria may produce many more molecules than was expected ${ }^{50}$. What is important to note is that this also applies to well-known families of molecules and in extensively studied model organisms. Examples are the highly rearranged cryptic polyketide lugdunomycin that belongs to the family of angucyclines ${ }^{51}$, the new glycopeptide corbomycin with a novel mode of action ${ }^{52}$, as well as the discovery of coelimycin ${ }^{53}$ and a novel branch of the actinorhodin biosynthetic pathway ${ }^{54}$ in the model organism Streptomyces coelicolor. The discovery of actinomycin 
L provides another interesting example that we have not yet exhausted the known part of the chemical space. Indeed, the isolation of these novel actinomycins underlines that the biosynthetic potential of Actinobacteria still has major surprises in store, and that we can expect that new molecules can be discovered even within extensively studied microbes and compound classes.

\section{Methods}

General experimental procedures. Optical rotation, FT-IR and UV were measured as previously described $^{55}$. NMR spectra were recorded on a Bruker Ascend $850 \mathrm{MHz}$ NMR spectrometer (Bruker BioSpin $\mathrm{GmbH}$ ). Data was analyzed using MestReNova 14 software (Mestrelab Research, Santiago de Compostela, Spain). The structures of molecules were drawn using ChemDraw Professional version 16.0 (Perkin-Elmer Informatics). HPLC purification was performed on Waters preparative HPLC system as described ${ }^{29}$. All solvents and chemicals were of HPLC or LC-MS grade, depending on the experiment.

Bacterial strains, growth conditions and metabolite extraction. Streptomyces sp. MBT27 was obtained from the Leiden University strain collection and had previously been isolated from the Qingling Mountains, Shanxi province, China ${ }^{28}$. Cultures were grown in triplicate in $100 \mathrm{~mL}$ Erlenmeyer flasks containing $30 \mathrm{~mL}$ of liquid minimal medium $(\mathrm{MM})^{56}$, supplemented with various carbon sources, and inoculated with $10 \mu \mathrm{L}$ of $10 \% / \mathrm{mL}$ spore suspension. The carbon sources (percentages in w/v) were: $1 \%$ mannitol $+1 \%$ glycerol, $1 \%$ mannitol, $2 \%$ mannitol, $1 \%$ glycerol, $2 \%$ glycerol, $1 \%$ glucose, $2 \%$ glucose, $1 \%$ fructose, $1 \%$ arabinose or $1 \%$ $\mathrm{N}$-acetylglucosamine (GlcNAc). The cultures were incubated in a rotary shaker at $30^{\circ} \mathrm{C}$ and $220 \mathrm{rpm}$ for seven days. Following fermentation, culture supernatants were extracted with ethyl acetate (EtOAc) and evaporated under reduced pressure. In the series of feeding experiments Streptomyces sp. MBT27, S. antibioticus IMRU 3720 and S. chrysomallus ATCC11523 were fermented in MM supplemented with $1 \%$ fructose and $0.7 \mathrm{mM}$ anthranilamide. For the directed biosynthesis of non-natural actinomycin X halves Streptomyces sp. MBT27 and S. antibioticus IMRU 3720 were grown in MM with $1 \% \mathrm{w} / \mathrm{v}$ fructose, $0.7 \mathrm{mM}$ anthranilamide and $0.7 \mathrm{mM}$ 3-hydroxy-4-methylbenzoic acid (4-MHB) and extracted with EtOAc.

Genome sequencing, assembly and annotation. Streptomyces sp. MBT27 was grown in YEME at $30^{\circ} \mathrm{C}$ and $220 \mathrm{rpm}$ for $48 \mathrm{~h}$. DNA was extracted from Streptomyces sp. MBT27 as described ${ }^{56}$. DNA quality was verified by agarose gel electrophoresis. PacBio sequencing and assembly was performed by Novogene (UK). Generally, library was prepared using SMRTbell template prep kit (PacBio, USA) according to manufacturer instructions. Sequencing was then performed using PacBio Sequel platform in continuous long reads mode. Assembly was done using Flye (version 2.8.1) ${ }^{57}$. Biosynthetic gene clusters (BGCs) in this genome were annotated using AntiSMASH 6.0 37 . The actinomycin BGC was then extracted and compared with the same cluster from S. antibioticus IMRU 3720 using clinker version 0.0.20) with default settings ${ }^{58}$.

Up-scale fermentation, extraction and fractionation. Large-scale fermentation, extraction end fractionation were performed as previously described ${ }^{29}$. The fractions eluted with $n$-hexane-acetone (1:1) was subjected to a SunFire $\mathrm{C}_{18}$ column $(10 \mu \mathrm{m}, 100 \AA$, $19 \times 150 \mathrm{~mm})$ eluted with a $\mathrm{H}_{2} \mathrm{O}-\mathrm{MeOH}$ gradient of $50-100 \%$ in $20 \mathrm{~min}$, at a flow rate of $15 \mathrm{~mL} / \mathrm{min}$. The fraction containing the actinomycins was collected and further purified on semi-preparative SunFire $\mathrm{C}_{18}$ column $(5 \mu \mathrm{m}, 100 \AA, 10 \times 250 \mathrm{~mm})$, run at $3 \mathrm{~mL} / \mathrm{min}$, and eluted using a $\mathrm{H}_{2} \mathrm{O}-\mathrm{MeOH}$ gradient of $70-100 \%$ in $20 \mathrm{~min}$, to yield actinomycins $\mathrm{L}_{1}(\mathbf{1}, 2.9 \mathrm{mg}), \mathrm{L}_{2}(2,1.3 \mathrm{mg}), \mathrm{X}_{2}(3,1 \mathrm{mg})$, $\mathrm{X}_{0 \beta}(4,2.9 \mathrm{mg})$, and $\mathrm{D}(5,3.2 \mathrm{mg})$.

Actinomycin $\mathrm{L}_{1}(\mathbf{1})$ : red amorphous powder; $[\alpha]_{\mathrm{D}}{ }^{25}-38$ (c 0.2, MeOH); UV (MeOH) $\lambda_{\max }(\log \varepsilon) 211(1.957)$, 312 (0.124), $427.5(0.152), 438(0.151) \mathrm{nm}$; IR $v_{\max } 3301,2963,2921,2859,1740,1662,1644,1585,1521,1465$, $1405,1300,1262,1191,1097 \mathrm{~cm}^{-1} ;{ }^{1} \mathrm{H}$ and ${ }^{13} \mathrm{C}$ NMR data, see Table 2; HRESIMS (positive mode) $\mathrm{m} / z 1387.6681$ $[\mathrm{M}+\mathrm{H}]^{+}$(calcd. for $\mathrm{C}_{69} \mathrm{H}_{91} \mathrm{~N}_{14} \mathrm{O}_{17}, 1387.6681$ ).

Actinomycin $\mathrm{L}_{2}$ (2): red amorphous powder; $[\alpha]_{\mathrm{D}}{ }^{25}-54(\mathrm{c} 0.2, \mathrm{MeOH}) ; \mathrm{UV}(\mathrm{MeOH}) \lambda_{\max }(\log \varepsilon) 226$ (2.189), $364(0.186) \mathrm{nm}, 426.5(0.367) \mathrm{nm}$; IR $v_{\max } 3308,2943,2929,2831,1748,1662,1644,1585,1448,1406,1302,1262$, $1191,1113 \mathrm{~cm}^{-1}$; HRESIMS (positive mode) $\mathrm{m} / z$ 1387.6674 $[\mathrm{M}+\mathrm{H}]^{+}$(calcd. for $\mathrm{C}_{69} \mathrm{H}_{91} \mathrm{~N}_{14} \mathrm{O}_{17}, 1387.6681$ ). The $\mathrm{X}$-ray diffraction experiment on a crystal grown from $\mathrm{MeOH}$ further confirmed the structure and determined the absolute configuration of compound 2 (Fig. 4) (CCDC 2,110,000). Structure visualization and image preparation was done using Mercury version 4.3.0

Antimicrobial activity assay and MIC determination. The antimicrobial activity of the compounds was tested in liquid inhibition assays against seven pathogens including Gram-negative and Gram-positive bacteria (Escherichia coli ATCC25922, Klebsiella pneumoniae ATCC700603, methicillin-resistant Staphylococcus aureus MB5393, methicillin-sensitive Staphylococcus aureus ATCC29213, linezolid-resistant Staphylococcus. epidermidis (clinical isolate), vancomycin-sensitive Enterococcus. faecium (clinical isolate), and vancomycinresistant Enterococcus faecium VanB (clinical isolate), as described ${ }^{60}$. Each compound was serially diluted in DMSO with a dilution factor of 2 to test 10 concentrations starting at $128 \mu \mathrm{g} / \mathrm{mL}$ in all the antimicrobial assays. The MIC was defined as the lowest concentration of compound that inhibited $\geq 95 \%$ of the growth of a microorganism after overnight incubation. The Genedata Screener software (Genedata, Inc., Basel, Switzerland) was used to process and analyze the data and to calculate the RZ' factor in the assay that was between 0.90 and 0.98 supporting its robustness. 


\begin{tabular}{|c|c|c|c|c|c|}
\hline Position & $\delta_{\mathrm{C}}$, type & $\delta_{\mathrm{H}}$, mult. $(J$ in $\mathrm{Hz})$ & Position & $\delta_{\mathrm{C}}$, type & $\delta_{\mathrm{H}}$, mult. $(J$ in $\mathrm{Hz})$ \\
\hline 1 & 129.5, C & & $22^{\prime}$ & 167.4, C & \\
\hline 2 & 130.9, C & & $23^{\prime}$ & 74.5, CH & $5.29, \mathrm{qd}(6.3,3.1)$ \\
\hline 3 & 125.9 & $7.75, \mathrm{~m}$ & $24^{\prime}$ & $17.5, \mathrm{CH}_{3}$ & $1.23, \mathrm{~d}(6.3)$ \\
\hline 4 & 130.0, CH & 7.44, dd $(7.6,0.9)$ & $25^{\prime}$ & 163.1, C & \\
\hline 5 & 128.5, C & & $26^{\prime}$ & 114.3, C & \\
\hline 6 & 140.6, C & & $27^{\prime}$ & 128.6, CH & $7.90, \mathrm{dd}(7.9,1.6)$ \\
\hline 7 & 144.7, C & & $28^{\prime}$ & 118.7, CH & $6.81, \mathrm{dt}(0.9,7.9)$ \\
\hline 8 & 113.6, C & & $29^{\prime}$ & 133.6, CH & 7.30, dt $(1.6,7.9)$ \\
\hline 9 & 178.9, C & & $30^{\prime}$ & 113.9, CH & $6.67, \mathrm{dd}(7.9,0.9)$ \\
\hline 10 & ND & & $31^{\prime}$ & 146.7, C & \\
\hline 11 & ND & & $1^{\prime \prime}$ & 166.4, C & \\
\hline 12 & ND & & $2 "$ & $55.5, \mathrm{CH}$ & $4.69, \mathrm{dd}(6.6,2.7)$ \\
\hline 13 & $15.1, \mathrm{CH}_{3}$ & $2.58, \mathrm{~d}(0.9)$ & NH-2" & & $7.64, \mathrm{~d}(6.6)$ \\
\hline 14 & $7.7, \mathrm{CH}_{3}$ & $2.26, \mathrm{~s}$ & $3^{\prime \prime}$ & 168.6, C & \\
\hline $1^{\prime}$ & 166.1, C & & $4^{\prime \prime}$ & 58.6, CH & $3.57, \mathrm{~m}$ \\
\hline $2^{\prime}$ & 54.7, CH & $4.45, \mathrm{dd}(6.4,3.1)$ & NH-4" & & $7.75, \mathrm{~m}$ \\
\hline NH-2' & & 7.38, d (6.4) & $5^{\prime \prime}$ & $31.8, \mathrm{CH}$ & $2.10, \mathrm{~m}$ \\
\hline $3^{\prime}$ & 168.7, C & & 6" & $18.9, \mathrm{CH}_{3}$ & $1.10, \mathrm{~d}(6.7)$ \\
\hline $4^{\prime}$ & 57.9, CH & $3.56, \mathrm{~m}$ & 7" & $19.2, \mathrm{CH}_{3}$ & $0.91, \mathrm{~d}(6.7)$ \\
\hline NH-4' & & $8.28, \mathrm{~d}(5.4)$ & $8^{\prime \prime}$ & ND & \\
\hline $5^{\prime}$ & 31.0, CH & $2.17, \mathrm{~m}$ & $9 "$ & $47.5, \mathrm{CH}_{2}$ & $\begin{array}{l}3.82, \mathrm{~m} \\
3.75, \mathrm{~m}\end{array}$ \\
\hline $6^{\prime}$ & $18.8, \mathrm{CH}_{3}$ & $1.13, \mathrm{~d}(6.7)$ & $10^{\prime \prime}$ & $22.8, \mathrm{CH}_{2}$ & $\begin{array}{l}2.29, \mathrm{~m} \\
2.07, \mathrm{~m}\end{array}$ \\
\hline $7^{\prime}$ & 19.1, $\mathrm{CH}_{3}$ & $0.87, \mathrm{~d}(6.7$ & $11^{\prime \prime}$ & 31.1, $\mathrm{CH}_{2}$ & $\begin{array}{l}2.84, \mathrm{~m} \\
1.86, \mathrm{dd}(11.9,6.9)\end{array}$ \\
\hline $8^{\prime}$ & ND & & $12^{\prime \prime}$ & $56.3, \mathrm{CH}$ & $5.95, \mathrm{~d}(9.2)$ \\
\hline $9^{\prime}$ & $60.5, \mathrm{CH}_{2}$ & $\begin{array}{l}4.49, \mathrm{~d}(13.4) \\
4.07, \mathrm{~d}(13.4)\end{array}$ & $13^{\prime \prime}$ & 172.9, C & \\
\hline $10^{\prime}$ & $76.8, \mathrm{C}$ & & $14^{\prime \prime}$ & $34.9, \mathrm{CH}_{3}$ & $2.89, \mathrm{~s}$ \\
\hline $11^{\prime}$ & $43.1, \mathrm{CH}_{2}$ & $\begin{array}{l}2.94, \mathrm{~m} \\
2.25, \mathrm{~m}\end{array}$ & $15^{\prime \prime}$ & $51.3, \mathrm{CH}_{2}$ & $\begin{array}{l}\text { 4.72, d (17.1) } \\
3.65, \mathrm{~d}(17.1)\end{array}$ \\
\hline $12^{\prime}$ & 56.7, CH & 6.27, d (10.5) & $16^{\prime \prime}$ & 165.4, C & \\
\hline $13^{\prime}$ & 173.1, C & & $17^{\prime \prime}$ & $39.4, \mathrm{CH}_{3}$ & $2.93, \mathrm{~s}$ \\
\hline $14^{\prime}$ & $35.1, \mathrm{CH}_{3}$ & $2.93, \mathrm{~s}$ & $18^{\prime \prime}$ & $71.4, \mathrm{CH}$ & $2.70, \mathrm{~m}$ \\
\hline $15^{\prime}$ & $51.1, \mathrm{CH}_{2}$ & $\begin{array}{l}\text { 4.36, d (17.1) } \\
\text { 3.58, d (17.1) }\end{array}$ & $19^{\prime \prime}$ & 27.0, $\mathrm{CH}$ & $2.69, \mathrm{~m}$ \\
\hline $16^{\prime}$ & 166.3, C & & $20^{\prime \prime}$ & $19.0, \mathrm{CH}_{3}$ & $0.75, \mathrm{~d}(6.3)$ \\
\hline $17^{\prime}$ & $39.1, \mathrm{CH}_{3}$ & $2.95, \mathrm{~s}$ & $21^{\prime \prime}$ & $21.7, \mathrm{CH}_{3}$ & $0.98, \mathrm{~d}(6.0)$ \\
\hline $18^{\prime}$ & 71.3, CH & $2.67, \mathrm{~m}$ & $22^{\prime \prime}$ & 167.2, C & \\
\hline $19^{\prime}$ & 26.9, CH & $2.65, \mathrm{~m}$ & $23^{\prime \prime}$ & $75.2, \mathrm{CH}$ & $5.22, \mathrm{qd}(6.3,2.7)$ \\
\hline $20^{\prime}$ & $19.0, \mathrm{CH}_{3}$ & $0.75, \mathrm{~d}(6.8)$ & $24^{\prime \prime}$ & $17.9, \mathrm{CH}_{3}$ & $1.30, \mathrm{~d}(6.3)$ \\
\hline $21^{\prime}$ & 21.6, $\mathrm{CH}_{3}$ & $0.96, \mathrm{~d}(6.4)$ & & & \\
\hline
\end{tabular}

Table 2. ${ }^{1} \mathrm{H}$ and ${ }^{13} \mathrm{C}$ NMR data of 1 in $\mathrm{CDCl}_{3}$ at $298 \mathrm{~K} .{ }^{1} \mathrm{H} 850 \mathrm{MHz}$ and ${ }^{13} \mathrm{C}$ NMR resonances inferred from HSQC and HMBC spectra.

LC-MS/MS analysis. For LC-MS analyses, extracts were dissolved in $\mathrm{MeOH}$ to a final concentration of $1 \mathrm{mg} / \mathrm{mL}$, and $1 \mu \mathrm{L}$ was injected into Waters Acquity UPLC system coupled to Agilent 6530 QTOF MS. Samples were analyzed according to the protocol that was previously published ${ }^{29}$. LC-MS/MS acquisition of the pure compounds was performed using Shimadzu Nexera X2 UHPLC system coupled to Shimadzu 9030 QTOF mass spectrometer as previously described ${ }^{61}$. LC-MS/MS acquisition for molecular networking was performed using Thermo Instruments MS system (LTQ Orbitrap XL, Bremen, Germany) equipped with an electrospray ionization source (ESI) as described ${ }^{29}$.

Computation of mass spectral networks. MS/MS raw data were converted to a 32-bit mzXML file using MSConvert (ProteoWizard) ${ }^{62}$ and spectral networks were assembled using Global Natural Product Social molecular networking (GNPS) (https://gnps.ucsd.edu) as described ${ }^{23}$. Briefly, the precursor ion mass tolerance was set to $2.0 \mathrm{Da}$ and a MS/MS fragment ion tolerance of $0.5 \mathrm{Da}$, while the minimum cosine score was set to 0.7. The data were clustered using MSCluster with a minimum cluster size of three spectra. The spectra in the 
network were also searched against GNPS spectral libraries. A minimum score of 0.7 was set for spectral library search, with at least six fragment peaks matching. Cytoscape 3.7.2 was used for visualization of the generated molecular networks ${ }^{32}$. In Cytoscape the detected chemical space is displayed as nodes and edges (nodes correspond to a specific consensus spectrum; edges represent significant pairwise alignment between nodes). The edge thickness was set to represent the cosine score, with thicker lines indicating higher similarity between nodes. LC-MS/MS data were deposited in the MassIVE Public GNPS data set (MSV000085106). The molecular networking job in GNPS can be found at https://gnps.ucsd.edu/ProteoSAFe/status.jsp?task=0c9153470404488 d8927289139f875d3. The annotated MS/MS spectra were deposited in the GNPS spectral library for actinomycin $\mathrm{L}_{1}$ (CCMSLIB00005718892) and $\mathrm{L}_{2}$ (CCMSLIB00005718891).

Statistical analysis. Prior to statistical analysis, mzXML files, which were converted using Shimadzu LabSolutions Postrun Analysis, were imported into Mzmine $2.31^{63}$ and processed as previously described ${ }^{29}$. The aligned peak list was exported as a comma-separated file for statistical analysis. Statistical analysis was performed using MetaboAnalyst ${ }^{64}$, where $\log$ transformation and pareto scaling was initially applied to the data. The normalized data were subjected to principal components analysis (PCA) and orthogonal partial least squares discriminant analysis (OPLS-DA). The quality of the models was evaluated with the relevant $R^{2}$ and $Q^{2}$. To identify the difference in intensity of a single mass feature among multiple growth conditions, one-way ANOVA was performed, followed by a post hoc Tukey's honest significant difference (HSD) test.

Received: 21 November 2021; Accepted: 1 February 2022

Published online: 18 February 2022

\section{References}

1. Wright, G. D. Solving the antibiotic crisis. ACS Infect. Dis. 1, 80-84 (2015).

2. Silver, L. L. Challenges of antibacterial discovery. Clin. Microbiol. Rev. 24, 71-109 (2011).

3. Barka, E. A. et al. Taxonomy, physiology, and natural products of the Actinobacteria. Microbiol. Mol. Biol. Rev. 80, 1-43 (2016).

4. Bérdy, J. Bioactive microbial metabolites. J. Antibiot. (Tokyo) 58, 1-26 (2005).

5. Bentley, S. D. et al. Complete genome sequence of the model actinomycete Streptomyces coelicolor A3(2). Nature 417, 141-147 (2002).

6. Cruz-Morales, P. et al. The genome sequence of Streptomyces lividans 66 reveals a novel tRNA-dependent peptide biosynthetic system within a metal-related genomic island. Genome Biol. Evol. 5, 1165-1175 (2013).

7. Ikeda, H. et al. Complete genome sequence and comparative analysis of the industrial microorganism Streptomyces avermitilis. Nat. Biotechnol. 21, 526-531 (2003).

8. Rutledge, P. J. \& Challis, G. L. Discovery of microbial natural products by activation of silent biosynthetic gene clusters. Nat. Rev. Microbiol. 13, 509-523 (2015).

9. van Bergeijk, D. A., Terlouw, B. R., Medema, M. H. \& van Wezel, G. P. Ecology and genomics of Actinobacteria: New concepts for natural product discovery. Nat. Rev. Microbiol. 18, 546-558 (2020).

10. Urem, M., Swiatek-Polatynska, M. A., Rigali, S. \& van Wezel, G. P. Intertwining nutrient-sensory networks and the control of antibiotic production in Streptomyces. Mol. Microbiol. 102, 183-195 (2016).

11. van der Heul, H. U., Bilyk, B. L., McDowall, K. J., Seipke, R. F. \& van Wezel, G. P. Regulation of antibiotic production in Actinobacteria: new perspectives from the post-genomic era. Nat. Prod. Rep. 35, 575-604 (2018).

12. Sanchez, S. et al. Carbon source regulation of antibiotic production. J. Antibiot. (Tokyo) 63, 442-459 (2010).

13. Bode, H. B., Bethe, B., Hofs, R. \& Zeeck, A. Big effects from small changes possible ways to explore nature's chemical diversity. ChemBioChem 3, 619-627 (2002).

14. Romano, S., Jackson, S. A., Patry, S. \& Dobson, A. D. W. Extending the "One Strain Many Compounds" (OSMAC) principle to marine microorganisms. Mar. Drugs 16, 244 (2018).

15. Yoon, V. \& Nodwell, J. R. Activating secondary metabolism with stress and chemicals. J. Ind. Microbiol. Biotechnol. 41, 415-424 (2014).

16. Zhu, H., Sandiford, S. K. \& van Wezel, G. P. Triggers and cues that activate antibiotic production by actinomycetes. J. Ind. Microbiol. Biotechnol. 41, 371-386 (2014).

17. Craney, A., Ozimok, C., Pimentel-Elardo, S. M., Capretta, A. \& Nodwell, J. R. Chemical perturbation of secondary metabolism demonstrates important links to primary metabolism. Chem. Biol. 19, 1020-1027 (2012).

18. Rigali, S. et al. Feast or famine: the global regulator DasR links nutrient stress to antibiotic production by Streptomyces. EMBO Rep. 9, 670-675 (2008).

19. Demarque, D. P. et al. Mass spectrometry-based metabolomics approach in the isolation of bioactive natural products. Sci. Rep. 10, $1051(2020)$.

20. Wu, C., Choi, Y. H. \& van Wezel, G. P. Metabolic profiling as a tool for prioritizing antimicrobial compounds. J. Ind. Microbiol. Biotechnol. 43, 299-312 (2016).

21. Gaudencio, S. P. \& Pereira, F. Dereplication: Racing to speed up the natural products discovery process. Nat. Prod. Rep. 32, 779-810 (2015).

22. Nguyen, D. D. et al. MS/MS networking guided analysis of molecule and gene cluster families. Proc. Natl. Acad. Sci. U. S. A. 110, E2611-2620 (2013).

23. Wang, M. et al. Sharing and community curation of mass spectrometry data with Global Natural Products Social Molecular Networking. Nat. Biotechnol. 34, 828-837 (2016).

24. Waksman, S. A. \& Woodruff, H. B. Bacteriostatic and bactericidal substances produced by a soil actinomyces. Proc. Soc. Exp. Biol. Med. 45, 609-614 (1940).

25. Katz, E. Actinomycin. (eds. D. Gottlieb \& P.D. Shaw) 276-341 (Springer, 1967).

26. Crnovcic, I. et al. Genetic interrelations in the actinomycin biosynthetic gene clusters of Streptomyces antibioticus IMRU 3720 and Streptomyces chrysomallus ATCC11523, producers of actinomycin X and actinomycin C. Adv. Appl. Bioinform. Chem. 2017, 29-46 (2017).

27. Crnovčić, I., Semsary, S., Vater, J. \& Keller, U. Biosynthetic rivalry of o-aminophenol-carboxylic acids initiates production of hemi-actinomycins in Streptomyces antibioticus. RSC Adv. 4, 5065-5074 (2014). 
28. Zhu, H. et al. Eliciting antibiotics active against the ESKAPE pathogens in a collection of actinomycetes isolated from mountain soils. Microbiology 160, 1714-1725 (2014).

29. Machushynets, N. V., Wu, C., Elsayed, S. S., Hankemeier, T. \& van Wezel, G. P. Discovery of novel glycerolated quinazolinones from Streptomyces sp. MBT27. J. Ind. Microbiol. Biotechnol. 46, 483-492 (2019).

30. Chong, J., Yamamoto, M. \& Xia, J. MetaboAnalystR 2.0: From raw spectra to biological insights. Metabolites 9, 57 (2019).

31. Laatsch, H. Antibase 2012, the natural compound identifier. (Wiley-VCH Verlag GmbH \& Co., 2012).

32. Shannon, P. et al. Cytoscape: A software environment for integrated models of biomolecular interaction networks. Genome Res. 13, 2498-2504 (2003).

33. Wang, Q. et al. Neo-actinomycins A and B, natural actinomycins bearing the $5 \mathrm{H}$-oxazolo[4,5-b]phenoxazine chromophore, from the marine-derived Streptomyces sp. IMB094. Sci. Rep. 7, 3591 (2017).

34. Semsary, S. et al. Ketonization of proline residues in the peptide chains of actinomycins by a 4-oxoproline synthase. ChemBioChem 19, 706-715 (2018).

35. Liu, M. et al. A systems approach using OSMAC, Log P and NMR fingerprinting: An approach to novelty. Synth. Syst. Biotechnol. 2, 276-286 (2017).

36. Keller, U. Acyl pentapeptide lactone synthesis in actinomycin-producing streptomycetes by feeding with structural analogs of 4-methyl-3-hydroxyanthranilic acid. J. Biol. Chem. 259, 8226-8231 (1984).

37. Blin, K. et al. antiSMASH 6.0: Improving cluster detection and comparison capabilities. Nucleic Acids Res. 49, W29-W35 (2021).

38. Jones, G. H. Actinomycin synthesis in Streptomyces antibioticus: Enzymatic conversion of 3-hydroxyanthranilic acid to 4-methyl3-hydroxyanthranilic acid. J. Bacteriol. 169, 5575-5578 (1987).

39. Pfennig, F., Schauwecker, F. \& Keller, U. Molecular characterization of the genes of actinomycin synthetase I and of a 4-methyl3-hydroxyanthranilic acid carrier protein involved in the assembly of the acylpeptide chain of actinomycin in Streptomyces. J. Biol. Chem. 274, 12508-12516 (1999).

40. Dewick, P. M. Medicinal Natural Products: A Biosynthetic Approach 3rd edn. (Wiley, 2009).

41. Shaaban, K. A. et al. Pyramidamycins A-D and 3-hydroxyquinoline-2-carboxamide; cytotoxic benzamides from Streptomyces sp. DGC1. J. Antibiot. (Tokyo) 65, 615-622 (2012).

42. Biabani, M. A. et al. Anthranilamides: new antimicroalgal active substances from a marine Streptomyces sp. J. Antibiot. (Tokyo) 51, 333-340 (1998).

43. Ham, Y. \& Kim, T.-J. Anthranilamide from Streptomyces spp. inhibited Xanthomonas oryzae biofilm formation without affecting cell growth. Appl. Biol. Chem. 61, 673-680 (2018).

44. Kolter, R. \& van Wezel, G. P. Goodbye to brute force in antibiotic discovery?. Nat. Microbiol. 1, 15020 (2016).

45. Katz, E., Pienta, P. \& Sivak, A. The role of nutrition in the synthesis of actinomycin. Appl. Microbiol. 6, 236-241 (1957).

46. Haque, S. F., Sen, S. K. \& Pal, S. C. Nutrient optimization for production of broad spectrum antibiotic by Streptomyces antibioticus SR15.4. Acta Microbiol. Immunol. Hung. 42, 155-162 (1995).

47. Theobald, U., Schimana, J. \& Fiedler, H. Microbial growth and production kinetics of Streptomyces antibioticus Tü 6040. Antonie Van Leeuwenhoek 78, 307-313 (2000).

48. Dalili, M. \& Chau, P. C. Production of actinomycin D with immobilized Streptomyces parvullus under nitrogen and carbon starvation conditions. Biotechnol. Lett. 10, 331-336 (1988).

49. Williams, W. K. \& Katz, E. Development of a chemically defined medium for the synthesis of actinomycin D by Streptomyces parvulus. Antimicrob. Agents Chemother. 11, 281 (1977).

50. Nett, M., Ikeda, H. \& Moore, B. S. Genomic basis for natural product biosynthetic diversity in the actinomycetes. Nat. Prod. Rep. 26, 1362-1384 (2009).

51. Wu, C. et al. Lugdunomycin, an angucycline-derived molecule with unprecedented chemical architecture. Angew. Chem. Int. Ed. Engl. 58, 2809-2814 (2019).

52. Culp, E. J. et al. Evolution-guided discovery of antibiotics that inhibit peptidoglycan remodelling. Nature 578, 582-587 (2020).

53. Gomez-Escribano, J. P. et al. Structure and biosynthesis of the unusual polyketide alkaloid coelimycin P1, a metabolic product of the cpk gene cluster of Streptomyces coelicolor M145. Chem. Sci. 3, 2716-2720 (2012).

54. Wu, C., Ichinose, K., Choi, Y. H. \& van Wezel, G. P. Aromatic polyketide GTRI-02 is a previously unidentified product of the act gene cluster in Streptomyces coelicolor A3(2). ChemBioChem 18, 1428-1434 (2017).

55. Elsayed, S. S. et al. Atypical spirotetronate polyketides identified in the underexplored genus Streptacidiphilus. J. Org. Chem. 85, 10648-10657 (2020).

56. Kieser, T., Bibb, M. J., Buttner, M. J., Chater, K. F. \& Hopwood, D. A. Practical Streptomyces Genetics (John Innes Foundation, 2000).

57. Kolmogorov, M., Yuan, J., Lin, L., Pevzner, P. A. Assembly of long error-prone reads using repeat graphs. Nat. Biotechnol. 37(5), 540-546. https://doi.org/10.1038/s41587-019-0072-8 (2019).

58. Gilchrist, C. L. M. \& Chooi, Y.-H. Clinker \& clustermap.js: Automatic generation of gene cluster comparison figures. Bioinformatics 37, 2473-2475 (2021).

59. Macrae, C. F. et al. Mercury 4.0: From visualization to analysis, design and prediction. J. Appl. Crystallogr. 53, 226-235 (2020).

60. Audoin, C. et al. Balibalosides, an original family of glucosylated sesterterpenes produced by the mediterranean sponge Oscarella balibaloi. Mar. Drugs 11, 1477-1489 (2013).

61. Xiao, X. et al. Functional and structural insights into a novel promiscuous ketoreductase of the lugdunomycin biosynthetic pathway. ACS Chem. Biol. 15, 2529-2538 (2020).

62. Chambers, M. C. et al. A cross-platform toolkit for mass spectrometry and proteomics. Nat. Biotechnol. 30, 918-920 (2012).

63. Pluskal, T., Castillo, S., Villar-Briones, A. \& Orešič, M. MZmine 2: Modular framework for processing, visualizing, and analyzing mass spectrometry-based molecular profile data. BMC Bioinform. 11, 395 (2010).

64. Chong, J. et al. MetaboAnalyst 4.0: Towards more transparent and integrative metabolomics analysis. Nucleic Acids Res. 46, W486-W494 (2018)

\section{Acknowledgements}

The work is supported by the NACTAR program of the Netherlands organization for Scientific Research (NWO), Grant No. 16440.

\section{Author contributions}

N.V.M. and G.P.v.W. conceived the work, N.V.M., S.S.E., C.D. and M.d.l.C. carried out the experiments and prepared the figures, M.A.S., O.G., T.H. and G.P.v.W. supervised the experiments and assisted in data interpretation, and N.V.M., S.S.E., C.D. and G.P.v.W. wrote the manuscript with the help of the other authors. All authors proofread the manuscript and agreed on the final version.

\section{Competing interests}

The authors declare no competing interests. 


\section{Additional information}

Supplementary Information The online version contains supplementary material available at https://doi.org/ 10.1038/s41598-022-06736-0.

Correspondence and requests for materials should be addressed to G.P.W.

Reprints and permissions information is available at www.nature.com/reprints.

Publisher's note Springer Nature remains neutral with regard to jurisdictional claims in published maps and institutional affiliations.

(c) (i) Open Access This article is licensed under a Creative Commons Attribution 4.0 International License, which permits use, sharing, adaptation, distribution and reproduction in any medium or format, as long as you give appropriate credit to the original author(s) and the source, provide a link to the Creative Commons licence, and indicate if changes were made. The images or other third party material in this article are included in the article's Creative Commons licence, unless indicated otherwise in a credit line to the material. If material is not included in the article's Creative Commons licence and your intended use is not permitted by statutory regulation or exceeds the permitted use, you will need to obtain permission directly from the copyright holder. To view a copy of this licence, visit http://creativecommons.org/licenses/by/4.0/.

(C) The Author(s) 2022 